\title{
Solitary tentorial sarcoid granuloma associated with Propionibacterium acnes infection: case report
}

\author{
Jiro Akimoto, MD, PhD, ${ }^{1}$ Kenta Nagai, MD, ${ }^{1}$ Daisuke Ogasawara, MD, ${ }^{1}$ Yujiro Tanaka, MD,, \\ Hitoshi Izawa, MD, ${ }^{1}$ Michihiro Kohno, MD, PhD, ${ }^{1}$ Keisuke Uchida, MT, ${ }^{2}$ and \\ Yoshinobu Eishi, MD, PhD² \\ 'Department of Neurosurgery, Tokyo Medical University; and 'Department of Human Pathology, Tokyo Medical and Dental \\ University Graduate School, Tokyo, Japan
}

\begin{abstract}
Sarcoidosis is a systemic granulomatous disease with unknown cause, which very rarely occurs exclusively in the central nervous system. The authors performed biopsy sampling of a mass that developed in the left tentorium cerebelli that appeared to be a malignant tumor. The mass was diagnosed as a sarcoid granuloma, which was confirmed with the onset of antibody reaction product against Propionibacterium acnes. Findings suggesting sarcoidosis to be an immune response to $P$. acnes infection have recently been reported, and they give insight for diagnosis and treatment of this disease. The authors report the possible first case that was confirmed with $P$. acnes infection in a meningeal lesion in solitary neurosarcoidosis.
\end{abstract}

https://thejns.org/doi/abs/10.3171/2016.8.JNS16480

KEY WORDS cerebellar tentorium; sarcoid granuloma; MRI; Propionibacterium acnes infection

$\mathrm{M}$ ORE than 130 years have passed since sarcoidosis was first identified, but the cause of this systemic granulomatous disease remains unknown. Sarcoidosis is designated as an intractable disease in Japan; the number of patients registered as having sarcoidosis exceeds 200,000. Mycobacterium tuberculosis infection has been suspected as a cause because clinical symptoms of sarcoidosis are similar to those of tuberculosis, but there has been no evidence of the presence of $M$. tuberculosis at the lesion site. Propionibacterium acnes, commensal skin bacteria, were isolated and cultured ${ }^{3,5-7}$ from the lymph nodes associated with lesions at a high frequency in Japanese studies conducted between 1978 and 1984. In addition, analysis using the antibody to a component of $P$. acnes ${ }^{10}$ suggested that $P$. acnes might cause sarcoidosis. We report a case associated with $P$. acnes infection in a solitary sarcoid granuloma that developed in the tentorium cerebelli.

\section{Case Report}

History and Examination

A 46-year-old woman presented with symptoms of dull headache and paresthesia on the left side of her face that lasted 6 months. She visited our department because she was gradually losing her hearing in the left ear. She had no notable medical or family history. Neurological findings showed moderate hypesthesia in the mandibular region of the left trigeminal nerve and hearing loss. No abnormalities were found on chest radiography or electrocardiography. Blood biochemistry test results were as follows: white blood cell count count $4900 / \mu l$, neutrophils $71.7 \%$, lymphocytes $22.3 \%$, low-density lipoprotein 149 U/L, Creactive protein $0.57 \mathrm{mg} / \mathrm{dl}$, anti-streptolysin $\mathrm{O} 55 \mathrm{IU} /$ $\mathrm{ml}$, negative for treponema pallidum latex agglutination, negative for HIV antigen, immunoglobulin G4 $38.8 \mathrm{mg} /$ $\mathrm{dl}$, angiotensin-converting enzyme $9.8 \mathrm{IU} / \mathrm{L} / 37^{\circ} \mathrm{C}$ (reference range $8.3-21.4 \mathrm{IU} / \mathrm{L} / 37^{\circ} \mathrm{C}$ ), negative for perinuclear anti-neutrophil cytoplasmic antibodies, negative for cytoplasmic anti-neutrophil cytoplasmic antibodies, and negative for QuantiFERON-TB gold. Cerebrospinal fluid examination showed mild inflammatory reaction with a mild increase in protein of $48 \mathrm{mg} / \mathrm{dl}$, a cell count of $17 /$ $\mu \mathrm{l}$ (mononuclear cells, $100 \%$ ), a $\beta 2$ microglobulin level of $1.84 \mathrm{mg} / \mathrm{L}$ (reference range $0.64-1.56 \mathrm{mg} / \mathrm{L}$ ), and a soluble interleukin-2 receptor antibody level of $92.4 \mathrm{U} / \mathrm{ml}$ (reference range $145-519 \mathrm{U} / \mathrm{ml})$.

ABBREVIATIONS PCR = polymerase chain reaction. 

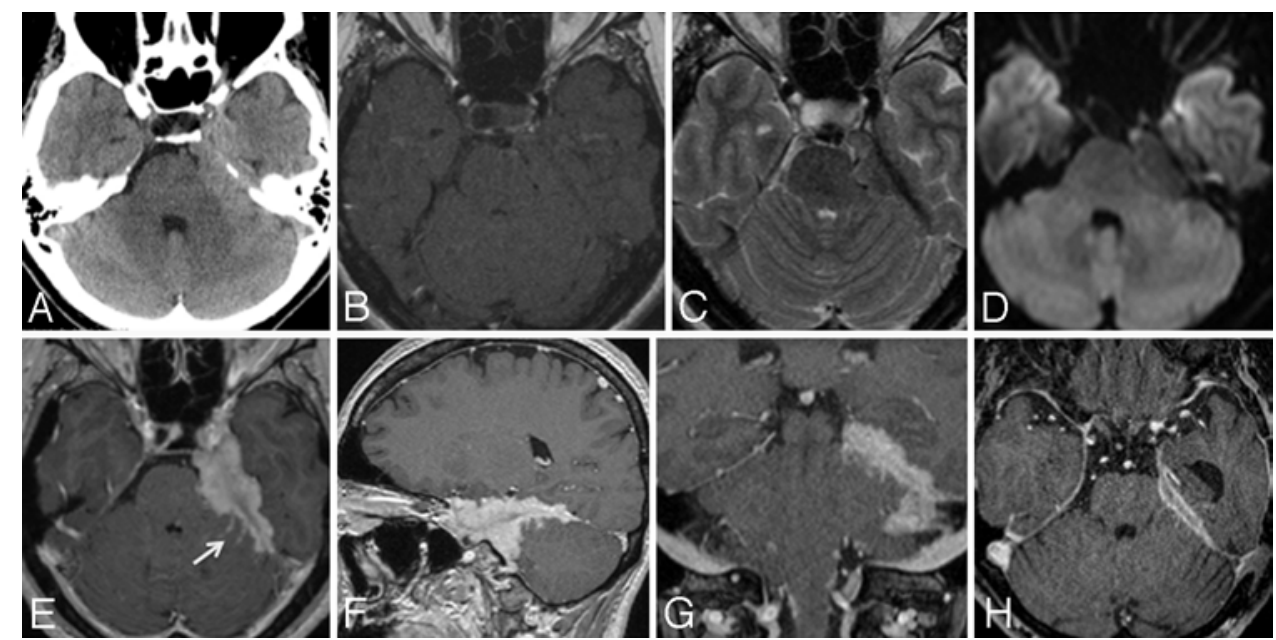

FIG. 1. A: Plain head CT scan obtained at admission to the hospital, revealing hypertrophy of the left tentorium cerebelli with slight density region. B: T1-weighted MR image showing en plaque mass of the iso-signal intensity in the left cerebellopontine angle cistern and mild mass effect in the left middle cerebellar peduncle. C: T2-weighted MR image showing the isointensity of the mass with the presence of a subarachnoid cavity between the mass and brainstem. A linear structure with low signal intensity was observed inside the mass along with the tentorium cerebelli. D: Diffusion-weighted MRI showing the iso-signal intensity of the mass. E: T1-weighted contrast-enhanced MR image showing a linear isointense band inside the mass, which irregularly extended to the cerebellum and the temporal lobe, and was homogeneously enhanced. A part of the band infiltrated into the cerebellar fissure (arrow), and homogeneously enhanced nodular lesions were observed in the contralateral peri-brainstem cisterns. $F$ and G: T1-weighted contrast-enhanced sagittal $(F)$ and coronal $(G)$ MR images revealing that the isointense band at the center of the mass indicated the tentorium cerebelli, and that the mass extended above and below from the tentorium cerebelli. H: T1-weighted contrast-enhanced MR image obtained at 1 year after prednisolone treatment and after biopsy, showing the internal auditory canal. Only mild enhancement in the surroundings of the dura mater was observed.

Head CT scanning revealed hypertrophy of the left tentorium cerebelli with a mild high-density region. MRI showed low signal on T1-weighted imaging and high signal on T2-weighted imaging at the same site, and there was a slit-like low signal band at the center of the site. Gadolinium-contrast-enhanced MR images showed homogeneous strong contrast at the site as well as infiltration into the cerebellar fissure and sporadic nodular lesions in the contralateral peri-brainstem cisterns. Cerebral angiography did not show obvious staining (Fig. 1A-G). For preoperative diagnosis, malignant meningioma, malignant lymphoma, metastatic lesion, or inflammatory disease such as hypertrophic pachymeningitis and Rosai-Dorfman disease were suggested.

\section{Operation}

Suspecting that the brain tumor might be malignant, tumor resection via a combined transpetrosal approach was attempted. Incision of the tentorium cerebelli revealed a whitish tumor lesion, which was elastic, fibrous, and slightly hard with slight bleeding. Based on an intraoperative diagnosis of inflammatory granulomatous lesion, only partial resection was conducted.

A diagnosis of sarcoid granuloma was made based on homogeneous granuloma tissues with infiltration of epithelioid cells mixed with Langhans giant cells and small lymphocytes in the absence of obvious caseous necrosis. Immunostaining with $P$. acnes antibody (PAB antibody, $P$. acnes-specific monoclonal antibody that reacts with cell membrane-bound lipoteichoic acid) showed clear granular staining areas in the cytoplasm of epithelioid cells other than Langhans giant cells (Fig. 2).

\section{Postoperative Course}

Hypesthesia of the left face was temporarily aggravated, but it gradually improved after methylprednisolone pulse therapy for 3 days followed by gradual decrease of the oral prednisolone dose from $60 \mathrm{mg}$ to $15 \mathrm{mg}$ and then maintaining the dose. However, hearing loss was not improved. Imaging findings showed gradual reduction of the tentorium cerebelli lesion, and the patient returned to her normal daily life (Fig. $1 \mathrm{H}$ ).

\section{Discussion}

A neurological lesion, neurosarcoidosis is observed in between $2.1 \%$ and $5 \%$ of patients with sarcoidosis. However, solitary manifestation of sarcoidosis in the central nervous system only accounts for about $1 \%{ }^{1,4}$ Clinical manifestations of sarcoidosis in the central nervous system are various, including meningeal lesions (meningitis, hypertrophic granular pachymeningitis), intracerebral granuloma, hydrocephalus, and vascular lesions (vasculitis, periventricular white matter lesions, sinus thrombosis), and sarcoidosis is characterized by the wide distribution of the disease. ${ }^{4}$ Carlson et al. ${ }^{1}$ analyzed 305 patients with cranial base neurosarcoidosis, and 117 patients (38\%) presented with solitary central nervous system lesions. Of these, 51 patients (17\%) had meningeal lesions, as observed in the present case, and the site of onset was near the internal auditory canal in 11 patients $(4 \%){ }^{1}$ In the present case the lesion was a solitary sarcoidosis of the cranial base without obvious lesions in the other organs. Imaging showed hypertrophic pachymeningitis, but it also showed findings of a meningitis type with infiltration into the peri- 

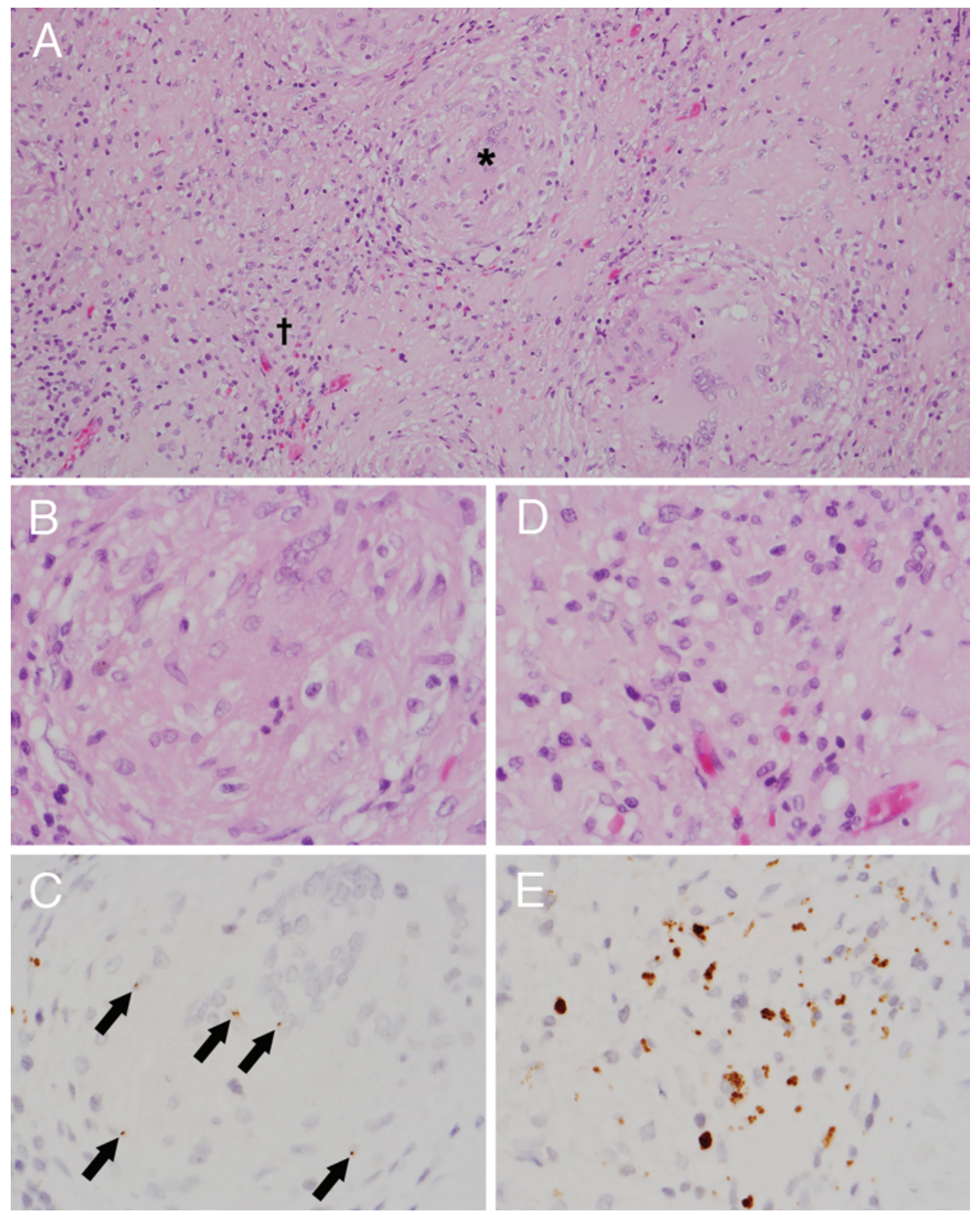

FIG. 2. Histopathological examination of the removed specimen and localization of $P$. acnes in granulomas. A: Multiple noncaseating epithelioid cell granulomas with hyalinization and fibrosis. B and C: A mature granuloma is seen (asterisk in A) with slight lymphocytic infiltration (B) and a few immunoreactive signals by the PAB antibody within the granuloma (C). D and E: An immature granuloma (cross in $A$ ) with much lymphocytic infiltration (D) and many immunoreactive signals scattered in clusters within the granuloma $(E)$. $H$ \& $E(A, B$, and $D) ; P A B$ antibody immunostain $(C$ and $E)$. Original magnification $\times 100(A) ; \times 400(B-E)$. Figure is available in color online only.

cerebellar fissure and nodular lesions in the contralateral peribrainstem cisterns.

A precise diagnostic method for neurosarcoidosis is not available, and the diagnosis rate is about $50 \%$ for increased angiotensin-converting enzyme level in the cerebrospinal fluid, which is conventionally considered useful. ${ }^{4} \mathrm{MRI}$ is thought to be the most effective imaging diagnostic method, although differentiation from meningeal tumor and inflammatory disease was difficult in the present case. Gallium scintigraphy and FDG (fluorodeoxyglucose)-PET were reported to be useful for diagnosis of sarcoidosis in other organs, but they are not useful for neurosarcoidosis.
Usefulness of FLT $\left({ }^{18} \mathrm{~F}\right.$-fluorothymidine)-PET has recently been reported, but biopsy appears indispensable for diagnosis of solitary sarcoidosis of the central nervous system. ${ }^{4}$

Although no therapeutic standard has been established, steroid treatment remains the first-line therapy and should be started as soon as possible. The dosing regimen is selected depending on the severity of the disease, and oral administration of prednisolone or pulse therapy with methylprednisolone is instituted. ${ }^{4}$ When a patient does not respond to steroid therapy, second-line therapy such as a combination of methotrexate and immunosuppressants, or administration of anti-tumor necrosis factor- $\alpha$ agent is 
attempted. However, no definite evidence has been established for the criteria of selecting drugs, dose, and maintenance period; the dosing regimen is selected at the discretion of the attending physician based on experience. ${ }^{4}$

One possible cause of sarcoidosis is allergic reaction following a preceding sensitization to a foreign substance. M. tuberculosis, fungi, nontuberculous mycobacteria, and viruses were examined as the cause, but verification was not possible. ${ }^{2}$ In 1999, Ishige et al. ${ }^{7}$ conducted polymerase chain reaction (PCR) analysis of the lymph nodes of sarcoidosis patients and detected DNA of P. acnes. In 2012, Negi et al. ${ }^{10}$ conducted tissue analysis using monoclonal antibody (PAB antibody) for L-form P. acnes cell membrane and confirmed an approximately $57 \%$ positive rate for the lung samples and $88 \%$ for the lymph nodes from sarcoid patients. ${ }^{10}$ Analysis using this antibody showed similar results, not only in Japanese but also in German patients. Furthermore, in a large-scale study of PCR analysis of lymph nodes in Italian and English patients, $P$. acnes DNA was detected in $98 \%$ of the patients. ${ }^{3}$ The above facts suggest that inapparent infection with $P$. acnes via the airway tract results in $P$. acnes remaining inactive in the lung and lung hilar lymph nodes prior to the onset of sarcoidosis. Then, granuloma is formed due to allergic reaction to proliferation of infectious $P$. acnes triggered by endogenous activation. ${ }^{2}$ Hematogenous dissemination of infectious $P$. acnes to the central nervous system is likely to cause the onset of lesions in other organs, such as the neurosarcoidosis observed in the present case. ${ }^{2,10}$

$P$. acnes is an obligate anaerobic, gram-positive bacillus indigenous in the skin and gastrointestinal tract and produces propionic acid, which is a saturated fatty acid..$^{27,10}$ The presence of $P$. acnes is usually monitored by the immune system because its excessive presence causes tissue toxicity. However, P. acnes is capable of escaping monitoring by the immune system by removing its cell wall. ${ }^{2,10} \mathrm{In}$ fact, P. acnes that was detected in Hamazaki-Wesenberg bodies found in the lymph nodes of sarcoidosis patients was reported to be the L-form without cell walls. ${ }^{2}$ Given that the anti-PAB antibody used in the present case is also a monoclonal antibody to L-form $P$. acnes, granular positive findings in the dural granuloma are likely to be a local allergic reaction to active infection by $\mathrm{L}$-form $P$. acnes that has escaped monitoring by the immune system. ${ }^{2,10}$

The above findings suggest that quantitative measurement of $P$. acnes DNA in the blood or cerebrospinal fluid by PCR will be useful for diagnosis of solitary neurosarcoidosis and that antibiotics for $P$. acnes will be effective for the treatment. In fact, minocycline and doxycycline have been reported to be effective for sarcoidosis in other organs ${ }^{8,9}$ Therefore, diagnosis and treatment of neurosarcoidosis may dramatically change in the future.

\section{Conclusions}

This is possibly the first case report of Propionibacterium acnes (L-form) infection confirmed in the tissues of sarcoidosis that developed in solitary granulomatous pachymeningitis. This report suggests that analysis of the pathological condition of $P$. acnes infection is effective for the diagnosis and treatment of neurosarcoidosis.

\section{Acknowledgments}

We are grateful to the medical editors from the Department of International Medical Communications of Tokyo Medical University for editing and reviewing the initial English manuscript.

\section{References}

1. Carlson ML, White JR Jr, Espahbodi M, Haynes DS, Driscoll CLW, Aksamit AJ, et al: Cranial base manifestations of neurosarcoidosis: a review of 305 patients. Otol Neurotol 36:156-166, 2015

2. Eishi Y: Etiologic link between sarcoidosis and Propionibacterium acnes. Respir Investig 51:56-68, 2013

3. Eishi Y, Suga M, Ishige I, Kobayashi D, Yamada T, Takemura T, et al: Quantitative analysis of mycobacterial and propionibacterial DNA in lymph nodes of Japanese and European patients with sarcoidosis. J Clin Microbiol 40:198-204, 2002

4. Hebel R, Dubaniewicz-Wybieralska M, Dubaniewicz A: Overview of neurosarcoidosis: recent advances. J Neurol 262:258-267, 2015

5. Hiramatsu J, Kataoka M, Nakata Y, Okazaki K, Tada S, Tanimoto M, et al: Propionibacterium acnes DNA detected in bronchoalveolar lavage cells from patients with sarcoidosis. Sarcoidosis Vasc Diffuse Lung Dis 20:197-203, 2003

6. Ishige I, Eishi Y, Takemura T, Kobayashi I, Nakata K, Tanaka I, et al: Propionibacterium acnes is the most common bacterium commensal in peripheral lung tissue and mediastinal lymph nodes from subjects without sarcoidosis. Sarcoidosis Vasc Diffuse Lung Dis 22:33-42, 2005

7. Ishige I, Usui Y, Takemura T, Eishi Y: Quantitative PCR of mycobacterial and propionibacterial DNA in lymph nodes of Japanese patients with sarcoidosis. Lancet 354:120-123, 1999

8. Marshall TG, Marshall FE: Sarcoidosis succumbs to antibiotics-implications for autoimmune disease. Autoimmun Rev 3:295-300, 2004

9. Miyazaki E, Ando M, Fukami T, Nureki S, Eishi Y, Kumamoto T: Minocycline for the treatment of sarcoidosis: is the mechanism of action immunomodulating or antimicrobial effect? Clin Rheumatol 27:1195-1197, 2008

10. Negi M, Takemura T, Guzman J, Uchida K, Furukawa A, Suzuki Y, et al: Localization of Propionibacterium acnes in granulomas supports a possible etiologic link between sarcoidosis and the bacterium. Mod Pathol 25:1284-1297, 2012

\section{Disclosures}

The authors report no conflict of interest concerning the materials or methods used in this study or the findings specified in this paper.

\section{Author Contributions}

Conception and design: Akimoto. Acquisition of data: Akimoto, Uchida, Eishi. Analysis and interpretation of data: Akimoto. Drafting the article: Akimoto. Critically revising the article: Akimoto, Kohno, Eishi. Reviewed submitted version of manuscript: Akimoto, Nagai, Ogasawara, Tanaka, Izawa, Kohno, Eishi. Approved the final version of the manuscript on behalf of all authors: Akimoto. Study supervision: Akimoto.

\section{Correspondence}

Jiro Akimoto, Department of Neurosurgery, Tokyo Medical University, 6-7-1 Nishi-Shinjuku, Shinjuku-ku, Tokyo 160-0023, Japan. email: jiroaki@gmail.com. 